\title{
Images of the month 3: Transient perivascular inflammation of the carotid artery syndrome
}

\author{
Authors: Philipp Jud, ${ }^{A}$ Georg Kangler, ${ }^{B}$ Paul Gressenberger, ${ }^{C}$ Rupert H Portugaller ${ }^{D}$ and Marianne Brodmann ${ }^{\mathrm{E}}$
}

KEYWORDS: carotid artery, vascular disease

DOI: 10.7861/clinmed.2021-0349

\section{Case presentation}

A 44-year-old man presented to the emergency department with a painful, palpable mass in the area of the right carotid triangle lasting for 1 week. He was otherwise healthy without any trauma or further otorhinolaryngological symptoms. Other than a pain at the right carotid triangle on pressure, physical and otorhinolaryngological examinations were unremarkable. Ultrasonography of the neck revealed a hypoechogenic mass in the adventitial layer of the right medial common carotid artery with a crescent shape in transverse view (Fig 1a) reaching from the distal common carotid artery to the carotid bulb $(35 \times 12 \times 6 \mathrm{~mm}$; Fig 1b). Carotid intima-media thickness was normal and no dissection membrane could be found. Subsequent ultrasonography of the aorta, its major branches and temporal arteries was also unremarkable, while magnetic resonance imaging of the carotid arteries revealed local contrast enhancement of the right common carotid artery (Fig 2). No potential signs or symptoms for inflammation, especially vasculitis, were found and comprehensive laboratory testing including inflammatory parameters, thyroid hormones, autoantibodies and immunoglobulin G4 was unremarkable. 18-fluorodeoxyglucose positron emission tomography - computed tomography showed only an increased tracer uptake of the right common carotid artery. Diagnosis of transient perivascular inflammation of the carotid artery (TIPIC) syndrome was made and dexibuprofen $400 \mathrm{mg}$ twice daily together with pantoprazole $40 \mathrm{mg}$ once daily peroral were prescribed for 2 weeks. The pain resolved within a few days and the hypoechogenic mass was undetectable in a followup visit 2 weeks later. Since the patient's initial presentation, he was still asymptomatic 1 year later.

Authors: ${ }^{\text {A }}$ registrar angiologist, Medical University of Graz, Graz, Austria; ${ }^{B}$ registrar otorhinolaryngologist, Medical University of Graz, Graz, Austria; ${ }^{C}$ registrar angiologist, Medical University of Graz, Graz, Austria; ${ }^{\mathrm{D}}$ consultant radiologist, Medical University of Graz, Graz, Austria; ${ }^{E}$ consultant angiologist, Medical University of Graz, Graz, Austria
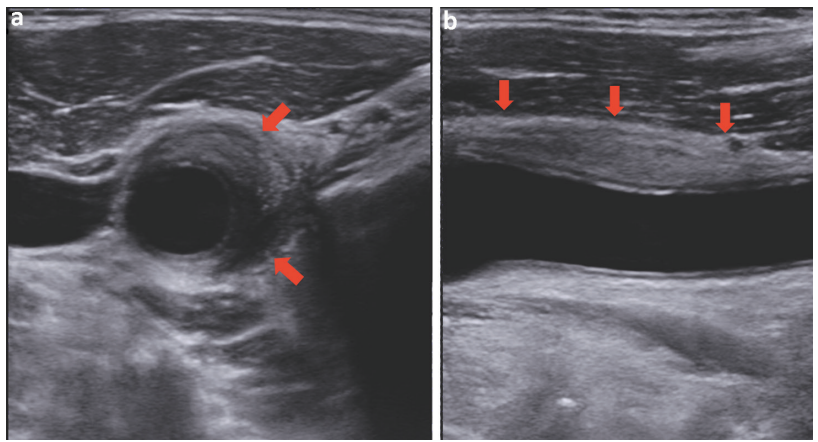

Fig 1. Ultrasonography of the neck showing a hypoechogenic mass in the adventitial layer of the right medial common carotid artery with a crescent shape in transverse view reaching from the distal common carotid artery to the carotid bulb.

\section{Discussion}

TIPIC syndrome, formerly known as idiopathic carotidynia, was firstly described by Fay in 1927 and recent data suggested that idiopathic carotidynia is a distinct disease due to imaging abnormalities of the carotid artery and introduced the term transient perivascular inflammation of the carotid artery syndrome.', 1,2 TIPIC syndrome is a rare, clinicoradiologic entity with crescent thickening of the carotid artery, but without affecting the blood flow or intima-media thickening. While its epidemiology and pathophysiology are unknown, it may be a kind
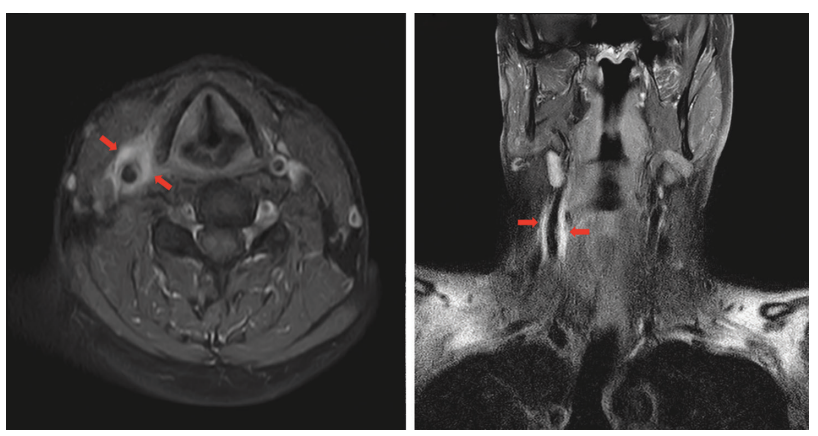

Fig 2. Magnetic resonance imaging of the carotid arteries showing local contrast enhancement of the right common carotid artery. 
of perivascular inflammation or vasculitis. ${ }^{2,3}$ Our patient fulfilled all proposed criteria for TIPIC syndrome and parainfectious processes with molecular mimicry contributing to vascular changes were suggested since the patient suffered from a sinusitis 2 weeks prior to presentation. TIPIC syndrome seems to be a frequently underdiagnosed disease and long-term outcome data of TIPIC syndrome are missing, further studies are needed to evaluate potential risks of this disease.

\section{References}

1 Fay T. Atypical neuralgia. Arch Neurol Psychiatry 1927;18: 309-15.
2 Lecler A, Obadia M, Savatovsky ] et al. TIPIC Syndrome: beyond the myth of carotidynia, a new distinct unclassified entity. Am J Neuroradiol 2017:38:1391-8.

3 Taniguchi $Y$, Horino T, Hashimoto K. Is carotidynia syndrome a subset of vasculitis? J Rheumatol 2008:35:1901-2.

Address for correspondence: Dr Philipp Jud, Division of Angiology, Department of Internal Medicine, Medical University of Graz, Auenbruggerplatz 15, $8036 \mathrm{Graz}$, Austria. Email: philipp.jud@medunigraz.at 